\title{
Susceptibility to geometrical illusions according to culture and professional training ${ }^{\prime}$
}

\begin{abstract}
This paper reports a cross-cultural investigation of susceptibility to geometrical illusions based upon student samples in Ghana and Scotland. The results indicate that: (1) cultural differences in susceptibility to illusions can be found among $S$ s exposed to lengthy formal education of a similar type; (2) the perceptual consequences of professional training in art and architecture tend to be moderate within a culture; but (3) the combined outcome of training across cultures tends to reduce cultural differences in susceptibility to illusions. They also raise a problem concerning the supposed positive relationship between field dependence and susceptibility to illusions. Some implications of these results are considered.
\end{abstract}

Interest in geometrical illusions dates back well over a century, but it still cannot be claimed that their nature is adequately understood. While there is no dearth of theories, as shown by Over's (1968) review, many of them suffer from serious weaknesses. Insofar as such theories aspire to generality, they are often based on studies (1) with too small a number and too homogeneous a group of Ss, (2) using too few illusions, and (3) confined to a Euroamerican setting. Regarding the last point, Over (1968) does scant justice to the cross-cultural research, which provides a potential touchstone for the range and power of a theory. For instance, physiological theories ought clearly to be tested cross-culturally, since it cannot be assumed that psychophysiological functioning remains unaffected by environmental influences (Biesheuvel, 1969); or again, certain theories, such as that of Piaget (1969), postulate relationships between different illusions that ought to hold cross-culturally; lastly, cultural differences in susceptibility themselves need explaining.

The most far-reaching investigation of the last problem is, of course, that of Segall et al (1966), who argued that geometric illusions are at least in part a function of perceptual habits of inference that possess ecological cue validity. Their data supported predictions that people living in industrialized or other "carpentered" environments would be most susceptible to the Müller-Lyer (ML) and the Sander parallelogram (SP) illusions, and that people living in vast, open terrains would show a greater response to the horizontal-vertical (HV) and combined horizontal-vertical-dichosection illusions. Other investigations tend to support the former prediction, but provide equivocal findings with regard to the latter (Berry, 1967; Dawson, 1967; Jahoda, 1966). However, Deregowski (1967) pointed out that the "foreshortening" hypothesis is only applicable to the HV effect and not to the dichosection (DS) effect, a contention borne out by the then-available data. Segall et al also administered the Poggendorff (PD) illusion but failed to obtain interpretable results.

While Segall et al analyzed their findings largely in terms of ecological variables, the study design was such that their results could also have been due to cultural variables and/or differences among the individuals comprising their samples. In order to clarify this issue, some investigations have been carried out within a single ethnic group living in an area with two distinct ecologies; this allows the comparison of ethnically and, in the main, culturally matched populations that have experienced different physical environments. Some of these studies provide at least limited support for the ecological hypothesis (Berry, 1968; Dawson, 1967), but others fail to do so (Berry, 1966; Gregor \& MacPherson, 1965; Jahoda, 1966). Berry (1968) has argued that nonsignificant findings from intracultural comparisons could be explained through the confounding of developmental and ecological variables. He demonstrated such a confounding effect within Eskimo and Temne (Sierra Leone) populations, showing that its elimination revealed the significance of both developmental (assessed in terms of the extent of field-dependence) and ecological variables on susceptibility to the ML illusion. Other investigators have claimed a positive relationship between field-dependence and susceptibility to certain illusions (Berry, 1967; Gardner, 1961 ; Pressey, 1967).

The present study, focusing on comparisons between Ss in Scotland and Ghana, is only secondarily concerned with the testing of specific hypotheses. It seeks to open up some broader issues that have so far received relatively little attention. The first of these issues is the effect of formal education, rightly regarded as a powerful modifier of psychological functioning (Greenfield \& Bruner, 1967; Irvine, 1969; Vernon, 1969). This variable remained uncontrolled in the Segall et al investigation and in some of the follow-up ones. The question may therefore be asked whether or not cultural differences in susceptibility to illusions can be found among Ss exposed to lengthy formal education of a similar type. If differences persist with at least some illusions, one can also consider the consequences of extended professional training in such fields as art or architecture that appears to be relevant to the making of perceptual judgments (Gombrich, 1959; Thouless, 1932). While it has been suggested that such a form of training is likely to reduce susceptibility to geometric illusions (Fraisse \& Vautrey, 1956; Segall et al, 1966), there is little evidence to support this suggestion.

The basic design of our study was, therefore, a simple fourfold one: Scottish vs Ghanaian students, and students untrained vs students trained in the fields of art and architecture. Since higher education in Ghana was introduced from Britain and follows closely similar lines in both countries, the factors of general education and specialized training can legitimately be treated as fairly uniform in both cultures. There are, of course, differences in ecology, to be mentioned shortly. Another feature of the design is the deployment of a wider range of illusions than have hitherto been used in this kind of study. This will permit the examination of relationships between various illusions and the extent to which these remain invariant across cultures. Such relationships are clearly relevant to the theoretical efforts to explain illusions.

While this is not a major purpose of the study, it is possible to formulate at least some tentative predictions on the basis of the ecological hypotheses of Segall et al concerning the ML, SP, and HV illusions. The physical environment of Ghanaian as well as Scottish students is definitely "carpentered," but the Ghanaians probably spent a substantial part of their childhood 


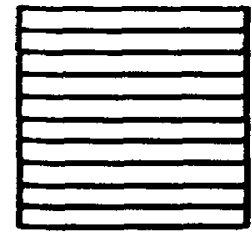

HELMHOLTZ SQUARE

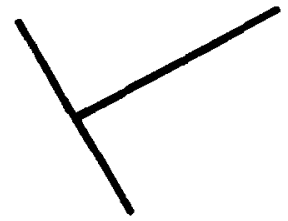

DICHOSECTION

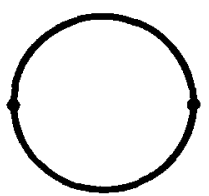
BORING CIRCLES

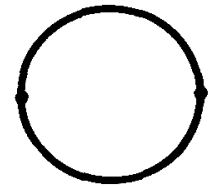

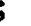

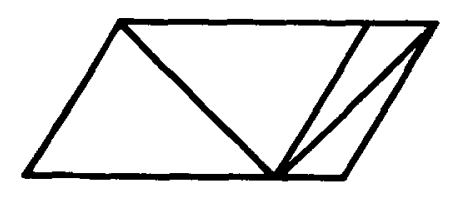

SANDER PARALLELOGRAM
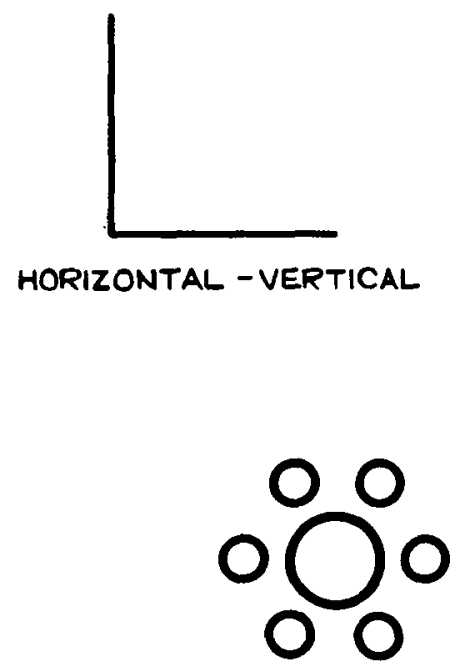

TITCHENER

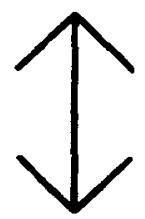

MÜLLER - LYER
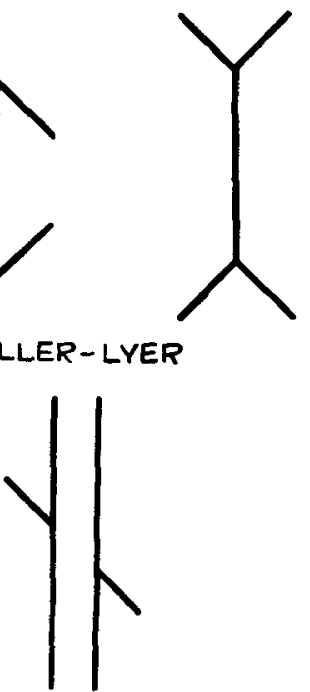

POGGENDORFF

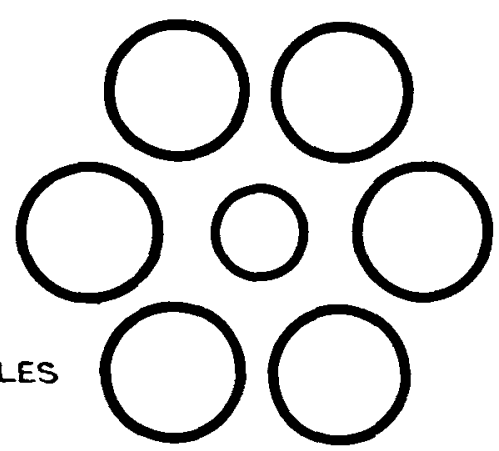

Fig. 1. Illusion Series.

in a somewhat less "carpentered" setting. Thus, on the assumption that the "carpentered" environment can be treated as a continuous variable, one would predict greater susceptibility of the Scots Ss to the ML and SP illusions. With regard to the "foreshortening" hypothesis, there are no open plains in Scotland, and, in any case, most of the Scottish Ss were city dwellers. In Ghana, a majority of the Ss came either from the coastal plains or the central forest belt. Since there is much geographical mobility among Ghanaian students, no clear division would be possible; but on balance one would expect the Ghanaian Ss to have a greater chance of having been exposed to open terrain, and thus predict their greater susceptibility to the HV illusion.

Finally, the study will touch upon the question of field-dependence, and the data collected permit a test of Deregowski's interpretation of the HV and DS illusions in a cross-cultural context.

\section{Materials}

\section{METHOD}

The primary criterion for the inclusion of specific illusions was their previous use in cross-cultural studies, i.e., in practice, mainly Segall et al. This accounts for the $\mathrm{ML}, \mathrm{SP}, \mathrm{PD}$, and $\mathrm{HV}$; because of the previously mentioned work of Deregowski, a right-angle form of the $\mathrm{HV}$ was employed and a dichosection (DS) figure added. The perspective illusion, found unsatisfactory by Segall et al, was omitted. In the absence of any generally accepted taxonomy of geometric illusions, the selection of the remaining ones was bound to be somewhat arbitrary, though governed by the restriction that they should range over the categories in the classificatory schemes of Boring (1942), Gregory (1966), and Oyama (1960).

The illusions used in the study are shown in Figs. 1 and 2; in the actual presentation, the lines to be judged were red, while any context features appeared in black. All the illusions in Fig. 1, except the Poggendorff, consisted of graded series of seven items: $15 \%, 10 \%$, and $5 \%$ below the standard, equal to the standard, and the same range above. In the case of PD, the series was made up of the following displacements of the bottom half of the oblique: $6,4,2 \mathrm{~mm}$ downwards, nil, and the corresponding displacements upwards; on the same scale, the length of the parallel lines was $65 \mathrm{~mm}$, and they were $10 \mathrm{~mm}$ apart. Perhaps a brief note on nomenclature should be inserted here. The now-common label of "Titchener Circles" (TC) has been adopted for the illusion originated by Ebbinghaus; the source of what we have called "Boring Circles" (BC) is not clear, but a version of it appeared in Boring, Langfeld, and Weld (1939).

The illusions in Fig. 2 are of a different kind and were presented only once, because the total length of the battery had to be kept within tolerable bounds. It does, of course, follow that for these, only group data could be meaningfully analyzed. In addition to the 60 main illusion items (eight sets of seven and four single ones), there were 14 others. Among these, two were control figures of pairs of vertical lines differing in length by $10 \%$, and two were control figures for the PD rotated by $45 \mathrm{deg}$. These control figures served as a rough check on the understanding of instructions, visual acuity, and the extent to which Ss remained alert throughout the testing session. Four items were mere buffers, designed to separate items in the sequence. Lastly, the pilot study had 


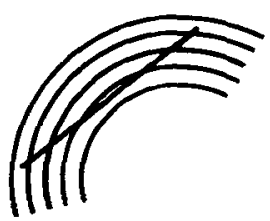

JUDO 1

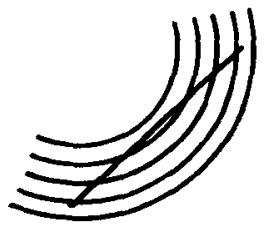

IUDD 2

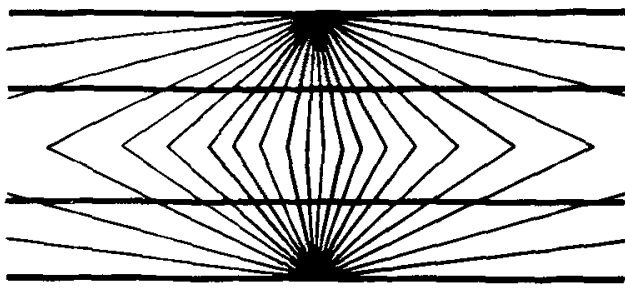

WUNDT

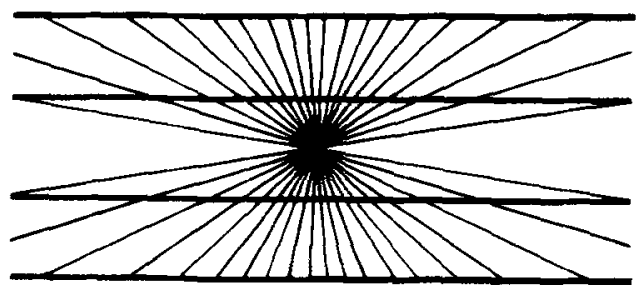

HERING

Fig. 2. Single Illusions,

shown the need to break the monotony if interest were to be maintained. For this purpose, six photographs of a girl were shown, Ss being asked to judge her emotional expression; this functioned as an effective periodic "arousal jag."

\section{Samples}

All Ss were students in universities, teacher training colleges, or similar institutions. An effort was made to obtain substantial numbers of $S s$ who either (1) lacked any experience of systematic instruction in drawing or related skills-other than is provided in the course of ordinary schooling-here called "untrained" (U), or (2) had at least two full years of such teaching, here designated as "trained" (T). A majority of the latter were students of art or architecture, while the former were teachers-in-training without any such specialization or students of languages, law, administration, or business. A total of 662 Ss were tested in four Scottish and six Ghanaian institutions, of whom 75 had to be eliminated subsequently. Apart from only two who failed on the control items, the basis for this elimination was information obtained in response to the question, "Please describe briefly any training you have received in drawing, painting, tracing, or design." Some of this information was inadequate or ambiguous, so that $\mathrm{Ss}$ could not be clearly assigned to the $U$ or $T$ categories; for instance, there were students who had at some stage in their career attended courses of unspecified length in technical drawing. The composition of the effective sample of 587
Ss was as follows: university students in Scotland-U 31, T 56; in Ghana-U 101, $\mathrm{T} 65$; students in training colleges and similar in Scotland-U 105, T 98; in Ghana-U 95, T 36. Although both $U$ and $T$ were adequately represented in each culture, there was some imbalance regarding their proportions and the types of institutions from which they were drawn. This was partly a function of differences in the educational systems, partly of differential access available. As a check on the possible effects of this, comparisons were made of $U$ and $T$ Ss, respectively, within different institutions in each culture, but no significant differences were encountered. There is, therefore, no indication that the divergencies in the institutional composition of the Scottish and Ghanaian samples affected the results.

\section{Procedures}

The stimulus materials were mounted on slides, and a Kodak Carousel projector controlled by the $\mathrm{E}$ was used. The conditions aimed at were a projected image size about $3 \mathrm{ft} 6 \mathrm{in}$. wide for a group-testing situation, with the first row of Ss no less than $8 \mathrm{ft}$ from the screen; moreover, groups of Ss were seated, as far as possible, near the center of the room to avoid too oblique an angle of vision. These conditions were closely approximated with all Scottish Ss and in Ghanaian universities. Some difficulties were experienced in Ghanaian colleges, especially with effective room darkening; in one case, these were not fully overcome so that a fair amount of ambient light was present. In Ghana, also, the intensive heat resulting from the darkening proved (except in one air-conditioned room) a pervasive problem, and towards the end of the procedure, lasting for about $1 \mathrm{~h}$, the room atmosphere was apt to be oppressive. Possibly this disturbed the $\mathrm{E}$ more than the $\mathrm{Ss}$, as there was fortunately no sign from internal comparisons that these unavoidable deficiencies had any bearing on performance.

The investigation was presented to the Ss as a cross-national study of visual perception. The core of the instructions ran like this: "You are going to be shown a series of drawings. Before the drawing appears on the screen, a question will be read out, and you can also find the same question in your booklet. Each drawing will be on view for 7 seconds. Immediately after the drawing has disappeared, you are asked to record your answer to the appropriate question by circling the A or B placed on the right of each question." Then followed a simple example of two lines differing by $20 \%$, with a further elaboration of the instructions. Three further examples were shown after this, the responses checked and questions invited. The actual administration did not begin until every member of the group of Ss seemed to have an adequate grasp of the nature of the task. All the items involved a simple binary choice of the type, "Which point is nearer to $X, A$ or $B$ ?" and neither Scottish nor Ghanaian Ss appeared to experience any difficulty in understanding what was required. This impression was subsequently borne out when the responses were analyzed.

\section{RESULTS}

The first step was to check whether or not the series had been functioning as intended, and this was confirmed by graphic plotting such as that shown in Figs. 3 and 4.

The basic findings are shown in Table 1. There were two significant intracultural differences in each sample, the untrained being more susceptible. In the cross-cultural comparisons between untrained Scottish and Ghanaian Ss, there were highly significant differences on all but the ML and DS illusions; and, with the exception of HS and HV, the Scots emerged as more susceptible than the Ghanaians. Within the subgroup of trained Ss, four of the differences disappeared, leaving only illusions to which Scottish Ss were more susceptible. A comparison of the responses of trained artists and trained architects produced no significant differences in susceptibility to any of the illusions.

In the case of the first eight illusions, it is possible to assign susceptibility scores 


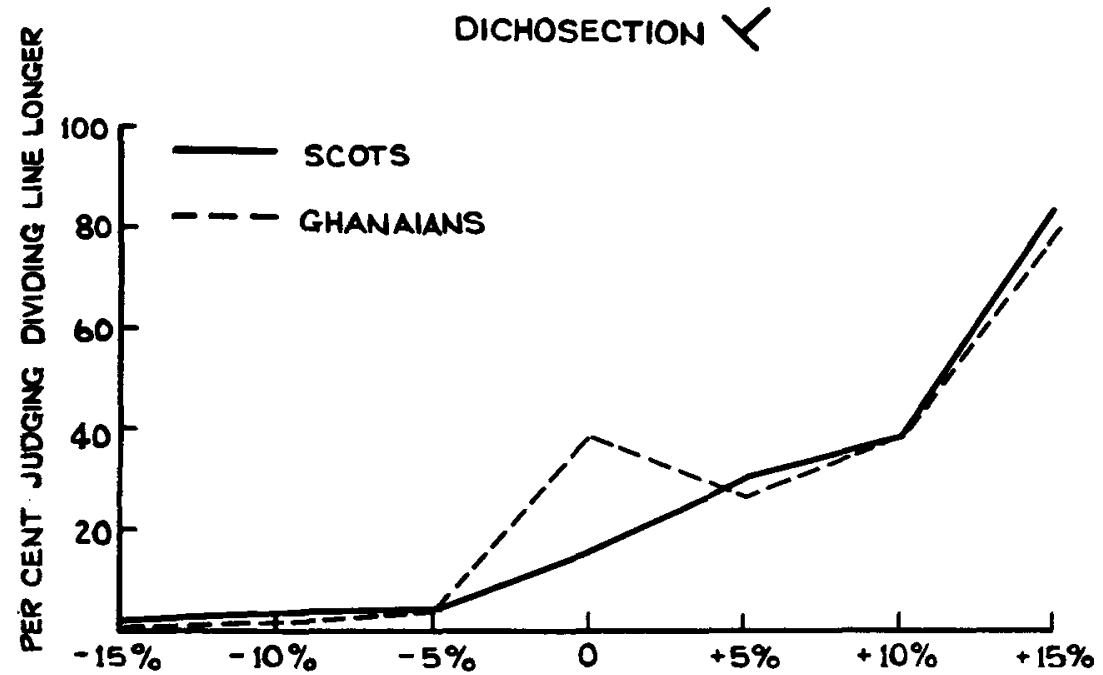

DIVIDING LINE IN RELATION TO DIVIDED LINE

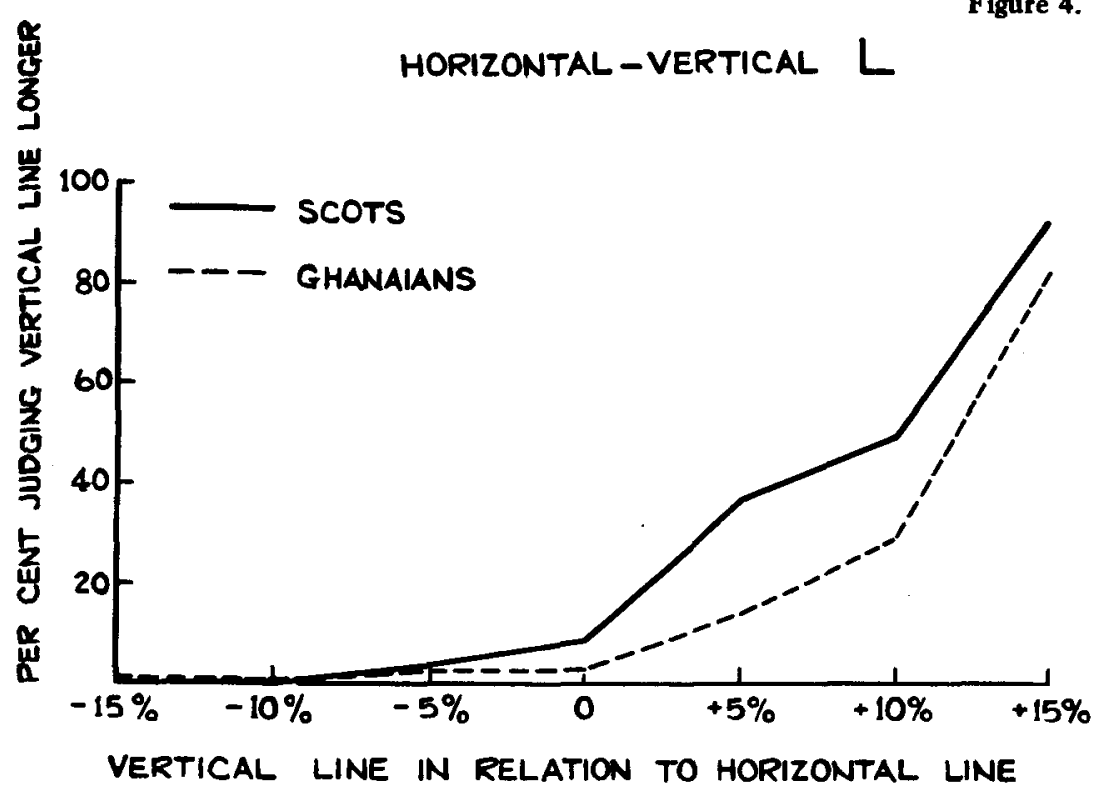

Table 1

Non-Parametric* Comparisons Between Untrained (U) and Trained (T) Scottish (S) and Ghanaian (G) Subjects (Greater susceptibility shown in brackets)

\begin{tabular}{|c|c|c|c|c|}
\hline Illusion & $\begin{array}{l}\text { Scottish } \\
\text { U vs T }\end{array}$ & $\begin{array}{c}\text { Ghanaian } \\
\text { U vs T }\end{array}$ & $\begin{array}{l}\text { Untrained } \\
\text { S vs G }\end{array}$ & $\begin{array}{l}\text { Trained } \\
\text { S vs G }\end{array}$ \\
\hline $\begin{array}{l}\text { Helmholtz Square } \\
\text { Sander Parallelogram } \\
\text { Múller-Lyer } \\
\text { Dichosection } \\
\text { Horizontal-Vertical } \\
\text { Poggendorff } \\
\text { Boring Circles } \\
\text { Titchener Circles } \\
\text { Judd } 1 \\
\text { Judd } 2 \\
\text { Hering } \\
\text { Wundt }\end{array}$ & $\begin{array}{l}\text { N.S. } \\
.001(U) \\
\text { N.S. } \\
\text { N.S. } \\
\text { N.S. } \\
\text { N.S. } \\
\text { N.S. } \\
\text {.025 (U) } \\
\text { N.S. } \\
\text { N.S. } \\
\text { N.S. } \\
\text { N.S. }\end{array}$ & $\begin{array}{l}.001(U) \\
\text { N.S. } \\
\text { N.S. } \\
\text { N.S. } \\
.001(U) \\
\text { N.S. } \\
\text { N.S. } \\
\text { N.S. } \\
\text { N.S. } \\
\text { N.S. } \\
\text { N.S. } \\
\text { N.S. }\end{array}$ & $\begin{array}{l}.001(G) \\
.001(S) \\
\text { N.S. } \\
\text { N.S. } \\
.001(G) \\
.001(S) \\
.001(S) \\
.001(S) \\
.001(S) \\
.001(S) \\
.001(S) \\
.001(S)\end{array}$ & $\begin{array}{l}\text { N.S. } \\
\text { N.S. } \\
\text { N.S. } \\
\text { N.S. } \\
\text { N.S. } \\
.001 \text { (S) } \\
\text { N.S. } \\
.001 \text { (S) } \\
.001(S) \\
.001 \text { (S) } \\
.01 \text { (S) } \\
.01 \text { (S) }\end{array}$ \\
\hline
\end{tabular}

- For the first eight illusions the Kolmogorov-Smirnov test was employed, and chi-squared for the remainder. from 0 (low) to 7 (high), and carry out a parametric analysis. Although the data in some cases fall short of normality and/or homogeneity of variance, the shape of the distributions always remains closely similar for the four subgroups. Hence, an analysis of variance was carried out as a subsidiary means of exploring the nature of the relationships involved. Some of these emerge very clearly from Table 2 . With the HS and SP illusions, the significant interactions indicate that training had the effect of bringing the odd subgroup out into line, and a weaker effect of this type occurs with the HV. One curious effect revealed in this table is that training appears to increase susceptibility to the BC illusion slightly among both Scottish and Ghanaian Ss. In general, this analysis yields an outcome that is quite consistent with the nonparametric one.

Another aspect of the data concerns the relationships between the first eight illusions; this is set out in the matrices of Table 3.

Four pairs of moderate but highly significant correlations are found to hold within both cultures: DS-ML, ML-SP, SP-HV, and HV-HS. In addition, there are a number of nonreciprocal correlations within each of the culture groups, four in the Scottish and eight in the Ghanaian sample. The PD stands out as not having a single significant correlation with any illusion in both samples. Cluster analysis of the Scottish matrix shows that the HS and HV illusions form a cluster, the SP, ML, and TC illusions, a second cluster. Principal components analysis of this matrix, with rotation to simple structure according to the varimax criterion, produces a first factor composed of the HS and HV illusions, and a second factor composed of the SP, ML, TC, and DS illusions. Cluster analysis of the Ghanaian matrix shows that the HS, HV, ML, and SP illusions form a general cluster. Principal components analysis with rotation produces three factors: the first composed of the SP, ML, and DS illusions, the second of the SP and TC, and the third a bipolar factor with the $\mathrm{HS}$ and $\mathrm{HV}$ at one end and the $\mathrm{BC}$ at the other.

A subsample of 65 untrained Ghanaians were administered the shortened version of the Embedded Figures Test. With one exception, nonsignificant correlations with illusion susceptibility were obtained; the exception was the PD, which showed an $r$ of -.30 . This means field-dependence was associated with lesser susceptibility.

\section{DISCUSSION}

The results relevant to the work of Segall et al will be considered first. While the cross-cultural comparisons on the SP 
Table 2

Sub-Group Means and Analysis of Variance by Culture and Training

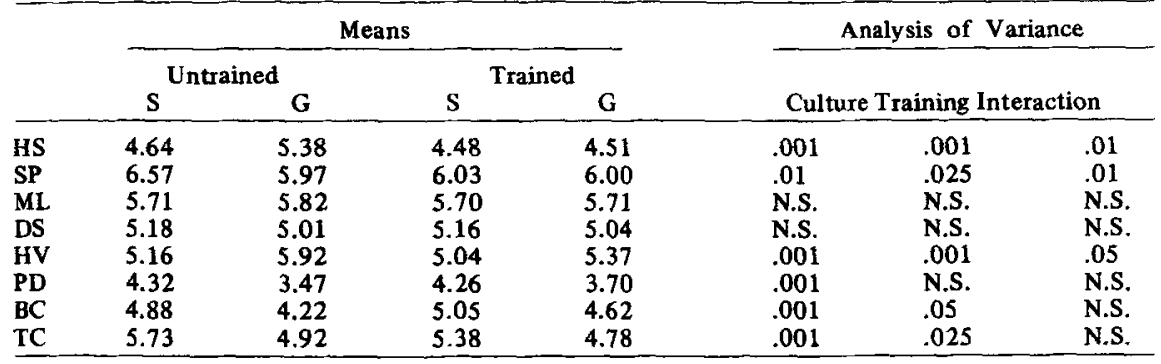

Table 3

Correlations Between Illusions Within Samples

\begin{tabular}{|c|c|c|c|c|c|c|c|c|}
\hline \multirow{2}{*}{$\begin{array}{l}\text { Scot- } \\
\text { land }\end{array}$} & \multicolumn{8}{|c|}{ Ghana } \\
\hline & HS & SP & ML & DS & HV & PD & $\mathrm{BC}$ & $\mathrm{TC}$ \\
\hline HS & & $19^{* * *}$ & $22^{* * *}$ & $13^{*}$ & $49 * * *$ & -01 & $-15^{*}$ & -01 \\
\hline SP & 02 & & $29 * * *$ & 11 & $26^{*} * *$ & -03 & 01 & $14 *$ \\
\hline ML & -05 & $23^{* * * *}$ & & $\underline{23} * * *$ & $\overline{17 * *}$ & -06 & -02 & $\overline{01}$ \\
\hline DS & 01 & $16^{* *} *$ & $22^{* * *}$ & & $15^{*}$ & -02 & 03 & -01 \\
\hline HV & $40^{* * *}$ & $21^{* * *}$ & $\overline{03}$ & 07 & & -08 & $-15^{*}$ & 05 \\
\hline PD & $-\overline{04}$ & $\overline{06}$ & 06 & 02 & -06 & & 00 & 01 \\
\hline BC & -02 & 03 & 07 & $15^{*}$ & 00 & -04 & & -06 \\
\hline TC & -05 & $25^{* * *}$ & $19^{* * *}$ & $17 * *$ & 05 & 04 & 02 & \\
\hline
\end{tabular}

** P less than .001; ** P less than .01; *P less than.05

Underlined coefficients indicate significant correlation in both cultures.

and ML illusions produced only a significant difference for the untrained on the SP, this could be interpreted as being not inconsistent with their hypothesis since both samples had experienced "carpentered" environments. They do suggest, however, that moderate differences in experience of a "carpentered" environment have a limited effect on susceptibility to the SP and ML illusions, as was previously indicated by Berry (1968) and Jahoda (1966). Since there is more flat, open terrain in Ghana, results on the HV illusion are in line with expectations based on the "foreshortening" hypothesis. Segall et al's prediction that artists and designers should be less susceptible to the SP and ML illusions, because they are less subject to constancy effects, is confirmed only for the SP in the Scottish sample.

Our findings throw some light on the perceptual consequences of professional training in art and architecture. Within cultures, these tend to be moderate. This may help to account for the fact that, while there has been a good deal of conjecture about the significance of special skills, few empirical results have been published; one recent study by Carlson (1966) found no significant effect. However, the combined outcome of training in the two different cultures was sufficient to eliminate highly significant cross-cultural differences in the cases of the HS, SP, HV, and BC illusions. With the BC, this occurred through a heightening of susceptibility consequent upon training, a explain in terms of current theories. One might speculate, for instance, that training intensifies the multiple perceptual activities that, according to Piaget, produce "secondary" illusion effects; but, if so, one would have expected similar heightening of susceptibility to the HS and HV illusions instead of the reduction that was found. It is also worth noting that apart from the problematic $B C$ illusion, the training effect was most marked in a set of illusions that tended to be significantly intercorrelated in both cultures. With the illusions on which there were no cross-cultural differences among the untrained (ML and DS), no PD was another special case, as no training effects were found in spite of powerful cross-cultural differences; and, as may be completely uncorrelated with any of the significant training effect, though not strong enough to obliterate the cross-cultural difference.

The results for the HV and DS illusions support Deregowski's contention that cross-cultural differences hold for the HV but not for the DS illusion. Deregowski's argument and reanalysis of available data seem to raise problems for Piaget's explanation of the HV illusion. Piaget attributes the perceptual errors found with the various HV figures to the way attention is controlled by the stimulus characteristics phenomenon that seems very difficult to discernable effect of training emerged. The recalled, this illusion was the only one rest. With the TC illusion, there was a of the figures. It is claimed that an observer attends to the top of a vertical and the middle of a horizontal. Movement from one fixation point to the other results in the vertical receiving a greater amount of attention, and for this reason it appears longer than a physically equal horizontal. Piaget also claims that the $\mathrm{HV}$ effect combines cumulatively with the dichosection effect in $T$ and $\perp$ figures. If this line of reasoning were correct, one would expect at least as marked cross-cultural differences for $T$ and 1 figures as for $L$ and $\Gamma$ figures, yet this is not the case. Further, it appears unlikely that the stimulus characteristics of HV figures would systematically influence acts of attention or, in Piagetian terms, the polarization of attentional explorations and transports in different ways in different cultures. It should be noted that Piaget's theoretical linking of the SP with the TC tends to be supported by the parts of our analysis relevant to these two illusions.

In considering the wider implications of these findings, a distinction has to be drawn between (1) the general determinants of particular illusion effects and (2) the factors responsible for the relative strength of an illusion within a specific cultural group; any obtained effect will always be a combined function of (1) and (2). Beginning with the latter, the results provide strong support for the contention by Segall et al that distinctive features of a culture lead to the formation of habits of perceptual inference that may enhance or inhibit susceptibility to particular illusions; whether these features be mainly ecological and/or involve child-rearing variables (a point to which it is proposed to return) cannot be decided without further study and probably varies with different illusions. What does emerge clearly is that such habits are modifiable by the influence of specialized training. Thus, the answer to one of the questions posed by Over, "Are illusions dependent on a subject's learning history?" is, "At least for some illusions, yes."

The wider implications of the present study can also be formulated in terms of another of Over's questions: "Do illusions constitute a single class of effects?" The substantial variations in the extent to which different illusions are affected by specialized training argues against this; and even stronger indication that the answer should be in the negative arises from the weak correlations between different illusion magnitudes and the fact that these are only partially consistent across cultures. But our data were collected in a way that did not allow us to test empirically Piaget's argument that 
geometric illusions can be classified in terms of their qualitative, but not their quantitative, characteristics.

One last issue arises from the finding that only one of our set of illusions was significantly related to field-dependence, and in a direction opposite to that found by other investigators in both industrial (Gardner, 1961; Immergluck, 1966; McGurk, 1965; Pressey, 1967) and nonindustrial cultures (Dawson, 1967; Berry, 1967). The last-named argued that child-rearing of a kind making for field-independence produces an analytical attitude entailing lower susceptibility to illusions; such ecological factors as "carpenteredness" may work in the other direction, leading to outcomes that vary with the relative strengths of such factors. In the present study, the magnitude of the PD illusion was greater with field-independent $S s$ in a Ghanaian untrained subsample. Since Ghanaians are generally far more field-dependent than Scots, and the latter were significantly more susceptible to the PD illusion, the present finding appears at least internally consistent. However, this contradiction does open up the problem of developmental influences on susceptibility to illusions, which is likely to be among the most fruitful next steps in advancing the understanding both of illusions in general and of cross-cultural differences in particular.

\section{REFERENCES}

BERRY, J. W. Temne and Eskimo perceptual skills. International Journal of Psychology, 1966, 1, 207-229.

BERRY, J. W. Independence and conformity in subsistence-level societies. Journal of Personality \& Socjaj Psychology, 1967, 7, 415-418.

BERRY, I. W. Ecology, perceptual development and the Müller-Lyer illusion. British Joumal of Psychology, 1968, 59, 205-210.

BIESHEUVEL, S. Methods for the measurement of psychological performance. Oxford: Blackwell, 1969.

BORING, E. G. Sensation and perception in the history of experimental psychology. New York: A ppleton-Century-Crofts, 1942.

BORING, E. G., LANGFELD, H. S., \& WELD, H. P. Introduction to psychology, New York: Wiley, 1939.

CARLSON, J. A. Effect of instructions and perspective-drawing ability on perceptual constancies and geometrical ilusions. Joumal of Experimental Psychology, 1966, 72, 874879 .

DAWSON, J, L. M. Cultural and physiological influences upon spatial-perceptual processes in West Africa. International Journal of Psychology, 1967, 2, 115-128 and 171-185.

DEREGOWSKI, J. The horizontal-vertical illusion and the ecological hypothesis. International Journal of Psychology, 1967, 2, 269-273.

FRAISSE, $P ., \&$ VAUTREY, P. The influence of age, sex, and specialized training on the vertical-horizontal illusion. Quarterly Journal of Experimental Psychology, 1956, 8, 114-120.

GARDNER, R. W. Cognitive controls of attention deployment as determinants of visual illusion. Journal of Abnormal \& Social Psychology, 1961, 62, 120-127.

GOMBRICH, E. H. Art and illusion. London: Phaidon, 1959.

GREENFIELD, P. M., \& BRUNER, J. Culture and cognitive growth. International Journal of Psychology, 1967, 1, 89-107.

GREGOR, A. J., \& McPHERSON, D. A. A study of susceptibility to geometric illusion among cultural subgroups of Australian aborigines. Psychologia Africana, 1965, 11, 1-13.

GREGORY, R. L. Visual illusions. In B. M. Foss (Ed.), New horizons in psychology. London: Penguin, 1966.
IMMERGLUCK, L. Resistance to an optical illusion, figural after-effects, and field dependence. Psyctonomic Science, 1966, 6, 281-282.

IRVINE, S. H, Factor analysis of African abilities and attainments: Constructs across cultures. Psychological Bulletin, 1969, 71, 20-32.

JAHODA, G. Geometric illusions and environment: A study in Ghana. British Joumal of Psychology, 1966, 57, 193-199.

McGURK, E. Susceptibility to visual illusions. Journal of Psychology, 1965, 61, 127-143.

OVER, R. Explanations of geometrical illusions. Psychological Bulletin, 1968, 70, 545-562.

OYAMA, T. Japanese studies on the so-called geometrical-optical illusions. Psychologia, $1960,3,7-20$.

PIAGET, J. The mechanisms of perception. London: Routledge, 1969.

PRESSEY, A. W. Field dependence and susceptibility to the Poggendorff illusion Perceptual \& Motor Skills, 1967, 24, 309-310.

SEGALL, M., CAMPBELL, D. T., \& HERSKOVITS, M. J. The influence of culture on visual perception. New York: Bobbs Merrill, 1966.

THOULESS, $R$. $H$. Individual differences in phenomenal regression. British Joumal of Psychology, 1932, 22, 216-241.

VERNON, P. E. Intelligence and cultural environment. London: Methuen, 1969.

\section{NOTES}

1. We should like to thank Dr. C. E. Fiscian and Mr. H. Bulley of Legon University for their assistance in planning the Ghana study, and to record our indebtedness to staff and students at institutions both in Ghana and Scotland for their generous cooperation. The support of the Nuffield Foundation, London, is also gratefully acknowledged.

2. Address: Department of Psychology, University of Strathclyde, Glasgow, C.1. Scotland.

(Accepted for publication August 8, 1969.) 